\title{
Decentralized Event-Based Communication Strategy on Leader-Follower Consensus Control
}

\author{
Duosi Xie, ${ }^{1}$ Xiaochun Yin, ${ }^{1}$ and Jianquan $X_{i e}{ }^{2}$ \\ ${ }^{1}$ School of Science, Nanjing University of Science and Technology, Nanjing 210094, China \\ ${ }^{2}$ Research Institute of Information Security, Hunan University of Finance and Economics, Changsha 410205, China
}

Correspondence should be addressed to Jianquan Xie; feiyingaizangma@126.com

Received 14 February 2016; Revised 12 June 2016; Accepted 28 June 2016

Academic Editor: Mirko Viroli

Copyright (c) 2016 Duosi Xie et al. This is an open access article distributed under the Creative Commons Attribution License, which permits unrestricted use, distribution, and reproduction in any medium, provided the original work is properly cited.

This paper addresses the leader-follower consensus problem of networked systems by using a decentralized event-based control strategy. The event-based control strategy makes the controllers of agents update at aperiodic event instants. Two decentralized event functions are designed to generate these event instants. In particular, the second event function only uses its own information and the neighbors' states at their latest event instants. By using this event function, no continuous communication among followers is required. As the followers only communicate at these discrete event instants, this strategy is able to save communication and to reduce channel occupation. It is analytically shown that the leader-follower networked system is able to reach consensus by utilizing the proposed control strategy. Simulation examples are shown to illustrate effectiveness of the proposed control strategy.

\section{Introduction}

Networked systems involve a large quantity of agents that cooperate with each other so that the system is able to complete sophisticated tasks [1-3]. As one of the networked systems, the leader-follower networked system has attracted a number of researchers to give contributions to coordinate control of the system [4-9].

Consider a physical networked system, where agents can only communicate with their local neighbors rather than all the other agents. In addition, the communication is subjected to limited bandwidths and the speed of wireless channels. In such a case, it would be preferable to develop a decentralized coordination control strategy that is able to accomplish the coordination goal with reduced communication occupation.

One of the strategies that fulfill this requirement is the socalled sampled-data control strategy [10-13]. In particular, a sampled-data control strategy, named event-based strategy, has attracted many attentions. The basic idea of the eventbased strategy is that the communication among agents only happen at irregular event instants [14-16]. Moreover, the actuator of the agents only updates at these irregular sampling instants as well. Therefore, the event-based strategy can also save updating energy, which makes the agents be able to achieve the same objectives even in situations with limited energy consumptions. These event instants are determined by predefined event functions. In a general decentralized eventbased strategy, each agent has its own event function which uses only the local information to calculate the agent's event instants. As a result, each agent has its own event sequence. Instead of using the continuous states of agents, the eventbased control law of each agent uses the agents' states sampled at their event instants.

So far, this strategy has been used on the coordination control of multiagent systems with different models [17, 18]. Meanwhile, different event-based communication and controller updating protocols have also been developed. The work in [18] proposes an event-based communication protocol such that each agent samples both its own and the neighbors' information at its own event instants. Another event-based strategy discussed in $[19,20]$ gives a different sampling scheme, where agents have synchronized sampling points with a fixed time interval. At each of these fixed points, each agent checks whether an event occurs using its event function. If so, the agent updates its controller and transmits information to its neighbors at this time instant. Otherwise the agent's controller remains unchanged and no information is transmitted until an event occurs. 
The event-based strategy has also been used to solve the consensus problem of multiagent systems with switching topologies [21, 22]. It is shown in [21] that a sufficient condition to guarantee the consensus is to have the topologies being balanced and strongly connected all the time. The work in [22] further relaxes the latter part of this sufficient condition and requires topology to be only jointly connected.

The event-based strategy has also been extended in solving the consensus problem of the so-called leader-follower networked systems [22-26]. In a leader-follower networked system, only part of the followers have access to the state of the leader. This would in general cause difficulties in the design of decentralized event functions. In [23, 24], a decentralized event-based control strategy is proposed to solve the leaderfollower consensus problem of a second-order networked system. However, the event function proposed therein has the limitation that the awareness of the leader's state is required to all the followers. The authors in [22, 26-29] propose different event-based control strategies to relax such a requirement. The authors in [22] solve the first-order leaderfollower consensus problem in the case where the topology is only jointly connected. The authors in [29] also consider the first-order leader-follower system. An event-based control strategy with combinational measurement error is proposed to solve the exponential synchronization problem of the system. Then the authors extended this method to general linear systems [25]. In [27], an event-based sampling strategy is developed such that each follower samples its own state, its neighbors' state, and the leader's state at the follower's event instants. Another sampling strategy proposed in [28] modifies this sampling scheme and let the follower be able to continuously receive the leader's state information.

As a large-scale leader-follower networked systems usually have only one leader but a large number of followers, the communication burdens are in general among the follower. Therefore, we aim at designing a proper event-based control strategy such that (1) the communication burdens among followers are reduced and (2) the number of controller updates is decreased. In this paper, a decentralized event-based control protocol and two event functions are proposed to solve the consensus problem in the leader-follower networked systems. Each follower updates its controller if any of its neighbors or itself has an event, which is further determined by their local predefined event function.

First of all, instead of using the most recent sampled state of neighbors, an event measurement is introduced in the event function to give an estimate of the states of neighbors. Secondly, the event function does not require a follower to be aware of the state of the leader if this follower does not have direct connection with the leader. Furthermore, the second event function does not require continuous states of neighbors to calculate the event instants. By using this event function, followers no longer need to continuously exchange information with their neighbors. Last but not least, the interevent intervals are analyzed in this work to exclude the possibility of Zeno-behavior (infinite accumulation of events in finite time).

The paper is organized as follows: Section 2 introduces some preliminaries and lemmas used later in this paper. The leader-follower consensus problem is also stated in Section 2. Section 3 addresses the main results of this paper, that is, the event-based control protocol with two event functions proposed for the leader-follower networked systems. Section 4 illustrates the effectiveness of the proposed control strategy using simulation examples. Section 5 concludes the paper.

Notation. Throughout this paper, $x^{T}$ and $\|x\|$ denote the transpose and the Euclidean norm of the vector $x$, respectively. For an arbitrary matrix $A, A^{T}$, and $\|A\|$ denote its transpose and 2-norm, respectively. For a symmetric matrix $A, \lambda_{\min }(A)$ and $\lambda_{\max }(A)$ represent the minimum and the maximum eigenvalue the matrix. $\mathbf{1}_{N} \in \mathbb{R}^{N}$ is a vector with all the entries being one. $I_{N}$ denotes an identity matrix with dimension $N$.

\section{Preliminaries}

2.1. Graph Theory. A graph $\mathscr{G}(\mathscr{V}, \mathscr{E})$ is used to describe the communication topology of a networked system, where $\mathscr{V}=$ $\{1,2, \ldots, N\}$ and $\mathscr{E} \subseteq \mathscr{V} \times \mathscr{V}$ are the set of nodes and edges, respectively. An agent $j$ is said to be the neighbor of agent $i$ if it is able to send information to agent $i$. This directed communication link is denoted as $(i, j) \in \mathscr{E}$. The set of all neighbors of agent $i$ is denoted by $N_{i}=\{j \mid(i, j) \in \mathscr{E}\}$. A topology is undirected if $(i, j) \in \mathscr{E}$ implies $(j, i) \in \mathscr{E}$. Otherwise the topology is directed. A path from agent $i$ to agent $k$ in $\mathscr{G}(\mathscr{V}, \mathscr{E})$ is a sequence of ordered edges. For an undirected graph, if there exist paths from every agent to every other agent, then the graph is connected. An adjacency matrix $\mathscr{A}=\left[a_{i j}\right] \in \mathbb{R}^{N \times N}$ is introduced to describe the graph topology, where the diagonal entries of $\mathscr{A}$ are zero $\left(a_{i i}=0\right.$, $\forall i=1, \ldots, N)$ and the off-diagonal entries $a_{i j}=1$, if $(j, i) \in \mathscr{E}$ and $a_{i j}=0$ otherwise. The Laplacian matrix $L=\left[l_{i j}\right] \in \mathbb{R}^{N \times N}$ is defined as $l_{i j}=-a_{i j}$ if $i \neq j$ and $l_{i i}=\sum_{j=1}^{N} a_{i j}$. Note that $L \mathbf{1}_{N}=0$. Moreover, if the topology is undirected, $A$ and $L$ are both symmetric and therefore, one also has $\mathbf{1}_{N}^{T} L=0$.

The leader-follower networked system considered in this paper contains $N$ followers and one leader. The topology among the followers is undirected and is described by graph $\mathscr{G}$ with its Laplacian matrix denoted as $L$. The leader can send information to followers, but not vice versa. Hence the topology that contains the leader and the followers is directed. A matrix $B=\operatorname{diag}\left\{d_{1}, d_{2}, \ldots, d_{N}\right\}$ is used to describe whether each follower directly receives information from the leader, where $d_{i}=1$ if follower $i$ can receive information from the leader directly, and $d_{i}=0$ otherwise.

The following lemmas state some properties of the Laplacian matrix used later in this paper.

Lemma 1 (see [30]). If the undirected graph $\mathscr{G}$ of a networked system is connected, then the Laplacian matrix of the graph has one zero eigenvalue and all the other eigenvalues are positive. Moreover, let $\lambda_{N}$ be the largest eigenvalue of the Laplacian matrix. Then for any vector $x \in \mathbb{R}^{N}$, one has $x^{T} L x \leq \lambda_{N} x^{T} x$.

Lemma 2 (see [31]). If the graph for the followers is connected and there exists at least one direct edge from the leader to a certain follower, then the matrix $L+B$ is positive definite. 
Lemma 3 (see [32]). Let $A_{m \times m}$ and $B_{m \times m}$ be Hermitian matrices. Then, $\lambda_{\text {min }}(A)+\lambda_{\text {min }}(B) \leq \lambda_{\text {min }}(A+B)$.

2.2. Problem Description. In this paper, we aim at solving the consensus problem of first-order leader-follower networked systems. The dynamics of the followers are

$$
\dot{x}_{i}(t)=b x_{i}(t)+u_{i}(t),
$$

where $x_{i}(t)$ is the position state of follower $i . b$ is a constant representing the inherent dynamics of the follower. $u_{i}(t)$ denotes the control law of the follower and is usually determined by the state of its neighbors. The dynamics of the leader is

$$
\dot{x}_{0}(t)=b x_{0}(t),
$$

where $x_{0}(t)$ denotes the position state of the leader. There is no control input for the leader; hence the leader will move following its inherent dynamics in (2).

Definition 4. The leader-follower networked system is said to achieve consensus if

$$
\lim _{t \rightarrow \infty}\left\|x_{0}(t)-x_{i}(t)\right\|=0, \quad \forall i=1, \ldots, N
$$

for any initial conditions.

A widely used control law to achieve consensus of the leader-follower networked system is

$$
\begin{aligned}
u_{i}(t)= & -k_{1} \sum_{j=1}^{N} a_{i j}\left(x_{i}(t)-x_{j}(t)\right) \\
& +k_{2} d_{i}\left(x_{0}(t)-x_{i}(t)\right),
\end{aligned}
$$

where $k_{1}$ and $k_{2}$ are positive scalar control gains. It is straightforward to see that this control law will update continuously. So are the communications among agents. As previously mentioned, we consider in the paper the case where the energy of the agent and the communication bandwidth are limited. When the agents carry multiple tasks while the coordination is only one of them, it is preferable to develop a coordination control strategy which reduces the energy consumed in both the controller updating and the communication channel occupation. By doing so, the agents can reserve enough energy and spare channel to accomplish other tasks.

The idea is to design an appropriate event function to monitor the state of the agents. When it is necessary, the event function generates an event. Only at this time instant will the agents sample and transmit their state and update their controller using the sampled states from their neighbors. Moreover, the event function should be designed in a way such that more events are generated when the system diverges and less events are generated when the system converges.

\section{Main Results}

In this section, the decentralized event-based control strategy is proposed to solve the consensus problem of leaderfollower networked systems while reducing the frequency of communication and local controller updates. The proposed strategy consists of event-based control law and decentralized event function. Each of the followers has its own event function which determines its own event time instants. As the event function is decentralized, no global communication is allowed. Using its local neighbors' state, each follower calculates its event instants and hence has its own event instant sequence. In this paper, we use $t_{1}^{i}, t_{2}^{i}, \ldots, t_{k}^{i}, \ldots$ to denote the event instant sequence of the follower $i$.

3.1. Consensus with Decentralized Event-Based Control Strategy. We first consider an approach which involves only neighbors' information in both the event function and the event-based control law of followers. For each follower, the control law is designed as

$$
u_{i}(t)=-k \sum_{j=1}^{N} a_{i j}\left(\tilde{x}_{i}(t)-\tilde{x}_{j}(t)\right)+d_{i}\left(x_{0}(t)-x_{i}(t)\right)
$$

where $k$ is a positive constant representing the control gain and $\tilde{x}_{i}(t) \triangleq x_{i}\left(t_{k}^{i}\right) e^{b\left(t-t_{k}^{i}\right)}, \forall i=1, \ldots, N, t \in\left[t_{k}^{i}, t_{k+1}^{i}\right)$, is referred as follower $i$ 's event measurement which is related to the event instant of the follower. Note that the design of $u_{i}(t)$ depends only on its own and its neighbors' event measurement. When the follower's event function reaches a certain threshold, the follower will update its event measurement with the latest event instant and the corresponding state at the instant. This event measurement will also be sent to the follower's neighbors in order to update their control laws.

Before stating the event function for each follower, we first define measurement error $\theta_{i}(t)$ for each follower: $\theta_{i}(t) \triangleq$ $x_{i}(t)-\tilde{x}_{i}(t), \forall i=1, \ldots, N$, that is, the difference between the current state of the follower and the event measurement. From the form of the event measurement defined in this paper, one has $\theta_{i}(t)=x_{i}(t)-x_{i}\left(t_{k}^{i}\right) e^{b\left(t-t_{k}^{i}\right)}, t \in\left[t_{k}^{i}, t_{k+1}^{i}\right)$, and one also has $\theta_{i}\left(t_{k}^{i}\right)=0$.

Therefore, with the defined $\theta_{i}(t)$, the follower's dynamics can be written as

$$
\begin{aligned}
\dot{x}_{i}(t)= & -k \sum_{j=1}^{N} a_{i j}\left(x_{i}(t)-x_{j}(t)-\theta_{i}(t)+\theta_{j}(t)\right) \\
& +d_{i}\left(x_{0}(t)-x_{i}(t)\right)+b x_{i}(t) .
\end{aligned}
$$

In this paper we use $\xi_{i}(t)$ to denote the state difference between the follower $i$ and the leader; that is, $\xi_{i}(t) \triangleq x_{0}(t)-$ $x_{i}(t)$. The dynamics of the leader-follower systems (1) and (2) can be written as

$$
\begin{aligned}
\dot{\xi}_{i}(t)= & b \xi_{i}(t) \\
& +k \sum_{j=1}^{N} a_{i j}\left(-\xi_{i}(t)+\xi_{j}(t)-\theta_{i}(t)+\theta_{j}(t)\right) \\
& -d_{i} \xi_{i}(t) .
\end{aligned}
$$


By synthesizing all $\xi_{i}$ into vector form and letting $H \triangleq$ $k L+B$ for simplicity, the above dynamics can be written into a compact form:

$$
\dot{\xi}(t)=b \xi(t)-k L \theta(t)-H \xi(t),
$$

where $\xi(t)=\left[\xi_{1}^{T}(t), \ldots, \xi_{N}^{T}(t)\right]^{T}$ and $\theta(t)=$ $\left[\theta_{1}^{T}(t), \ldots, \theta_{N}^{T}(t)\right]^{T}$. The decentralized event function $f_{i}(t)$ for each follower is designed as

$$
f_{i}(t)=\left\|\theta_{i}(t)\right\|-\sqrt{\frac{\sigma \alpha}{\beta}}\left\|z_{i}(t)\right\|,
$$

where $\sigma$ satisfies $0<\sigma<1$ and $\alpha$ and $\beta$ are both positive constants to be discussed later. $z_{i}(t)$ is the $i$ th element of the vector $z(t) \triangleq H \xi$. Note that if follower $i$ is not directly related to the leader, $z_{i}(t)$ depends only on the follower i's neighbors' states.

An event of a certain follower $i$ is generated when the event function $f_{i}(t)$ is greater than 0 . This instant will be marked as the latest event instant of the follower $i$. Without loss of generality, we use $t_{k}^{i}$ to denote this instant. As $\theta_{i}\left(t_{k}^{i}\right)=0$ by definition, one has $f_{i}\left(t_{k}^{i}\right) \leq 0$. After $t_{k}^{i},\left\|\theta_{i}(t)\right\|$ will fluctuate until the event function overpasses 0 again. Hence the event function $f_{i}(t)$ is always no greater than zero except for the left instant of the event time.

For a certain follower $i$, during the time intervals between its two consecutive event instants, if any of its neighbors is triggered, its control law will be updated with the most recently received neighbor state $x_{j}\left(t_{k^{j}}^{j}\right)$ and the instant $t_{k^{j}}^{j}$, $\forall j \in N_{i}$ (here $t_{k^{j}}^{j}$ denotes the latest event instant of follower j).

Theorem 5. Consider the leader-follower networked system described in (1) and (2). Suppose that the topology among followers is connected and at least one follower which can receive information from the leader. The followers use the control law (5) and the events of followers occur when the event function (9) overpasses zero. Then the consensus of the system can be achieved, if the following conditions are fulfilled:

$$
\begin{aligned}
b I_{N}-H & <0, \\
\frac{k k_{1}\|L\|}{2}-\tilde{\lambda} & <0,
\end{aligned}
$$

where $k_{1}$ is a positive constant and $\tilde{\lambda} \triangleq \lambda_{\text {min }}\left(H-b I_{N}\right)$.

Proof. Consider the following Lyapunov candidate:

$$
V(t)=\frac{1}{2} \xi^{T}(t) \xi(t) .
$$

The right derivative of $V(t)$ along the trajectory of (8) with respect to $t$ is

$$
\begin{aligned}
\dot{V} & =\xi^{T}\left(\left(b I_{N}-H\right) \xi-k L \theta\right) \leq-\tilde{\lambda} \xi^{T} \xi-\xi^{T} k L \theta \\
& \leq-\left(\tilde{\lambda}-\frac{k k_{1}\|L\|}{2}\right) \xi^{T} \xi+\frac{k\|L\|}{2 k_{1}} \theta^{T} \theta
\end{aligned}
$$

where the time $t$ is omitted for simplicity. The last line of (13) is due to the condition in (10) and Yang's inequality: $x^{T} y \leq$ $\left(k_{1} / 2\right) x^{T} x+\left(1 / 2 k_{1}\right) y^{T} y$ for any positive constant $k_{1}$.

From Lemma $2, H$ is positive definite. As $z=H \xi$, one has $\xi=H^{-1} z$. Note that $H^{-1}$ is also positive definite. It follows from (13) that

$$
\begin{aligned}
\dot{V} \leq & -\left(\tilde{\lambda}-\frac{k k_{1}\|L\|}{2}\right) z^{T}\left(H^{-1}\right)^{T}\left(H^{-1}\right) z \\
& +\frac{k\|L\|}{2 k_{1}} \theta^{T} \theta \\
\leq & -\left(\tilde{\lambda}-\frac{k k_{1}\|L\|}{2}\right)\left(\lambda_{\min }\left(H^{-1}\right)\right)^{2} z^{T} z \\
& +\frac{k\|L\|}{2 k_{1}} \theta^{T} \theta .
\end{aligned}
$$

By condition (11), one has $\left(\widetilde{\lambda}-k k_{1}\|L\| / 2\right)\left(\lambda_{\min }\left(H^{-1}\right)\right)^{2}>0$.

The coefficients $\alpha$ and $\beta$ in the event function are set into $\alpha=\left(\widetilde{\lambda}-k k_{1}\|L\| / 2\right)\left(\lambda_{\min }\left(H^{-1}\right)\right)^{2}$ and $\beta=k\|L\| / 2 k_{1}$. The event strategy guarantees that $\left\|\theta_{i}(t)\right\| \leq \sqrt{\sigma_{i} \alpha / \beta}\left\|z_{i}(t)\right\|$. Otherwise the follower will be triggered and hence will guarantee this inequality to hold. As a result, one has

$$
\dot{V} \leq \alpha(\sigma-1)\|z\|^{2} .
$$

Since $0<\sigma<1, \dot{V}$ is negative when the consensus is not achieved and is equal to zero when the consensus is achieved. Therefore, by the invariance principle, the followers will converge to the consensus space; that is, $x_{1}=x_{2}=\cdots=$ $x_{N}=x_{0}$. This concludes the proof.

It is well known that the existence of the Zeno-behavior must be excluded in the work of event-based control strategy. In other words, for each follower $i, i=1, \ldots, N$ in the system, the time interval between every two consecutive events must be strictly positive. We propose the following theorem to exclude the Zeno-behavior in our work.

Theorem 6. Consider the leader-follower networked system described in (1) and (2). Suppose that the topology among followers is connected and there exists at least one follower which can receive information from the leader. Also suppose that conditions (10) and (11) are satisfied. The followers are under control of law (5) and the events of followers occur when the event function (9) overpasses zero. Then the event intervals are strictly positive if the consensus is not achieved.

Proof. The event intervals can be obtained by analyzing $\theta_{i}(t)=x_{i}(t)-x_{i}\left(t_{k}^{i}\right) e^{b\left(t-t_{k}^{i}\right)}$. For follower $i$, at each event instant $t_{k}^{i}$, one has $\theta_{i}\left(t_{k}^{i}\right)=0$. From the event function, the $(k+1)$ th event instant is the time when $\left\|\theta_{i}(t)\right\|$ exceeds $\sqrt{\sigma \alpha / \beta}\left\|z_{i}(t)\right\|$. Thus, the time interval between the $k$ th and the $(k+1)$ th event is equal or longer than the time for $\left\|\theta_{i}(t)\right\|$ to increase from 0 to $\sqrt{\sigma \alpha / \beta}\left\|z_{i}(t)\right\|$. 
The dynamics of the measurement error $\theta_{i}(t)$ is

$$
\begin{aligned}
\theta_{i}(t) & =\theta_{i}\left(t_{k}^{i}\right)+\int_{t_{k}^{i}}^{t} \dot{\theta}_{i}(s) d s \\
& =\int_{t_{k}^{i}}^{t}\left(\dot{x}_{i}(s)-b x_{i}\left(t_{k}^{i}\right) e^{b\left(s-t_{k}^{i}\right)}\right) d s .
\end{aligned}
$$

By substituting the dynamics of follower $i$ from (6), it follows that

$$
\begin{aligned}
& \theta_{i}(t)=\int_{t_{k}^{i}}^{t}\left(-k \sum_{j=1}^{N} a_{i j}\left(\theta_{j}(s)-\theta_{i}(s)+\xi_{i}(s)-\xi_{j}(s)\right)\right. \\
& \left.+d_{i} \xi_{i}(s)-b x_{i}\left(t_{k}^{i}\right) e^{b\left(s-t_{k}^{i}\right)}\right) d s \\
& =\int_{t_{k}^{i}}^{t}\left(k L_{i} \theta(s)+k L_{i} \xi(s)+B_{i} \xi(s)\right. \\
& \left.+b I_{N i} \theta(s)\right) d s,
\end{aligned}
$$

where $L_{i}, B_{i}$, and $I_{N}^{i}$ denote the $i$ th row of the matrix, respectively, $L, B$, and $I_{N}$. Therefore, $\left(k L_{i}+B_{i}\right)$ is the $i$ th row of $H$. It follows that

$$
\begin{aligned}
\theta_{i}(t) & =\int_{t_{k}^{i}}^{t}\left(z_{i}(s)+\left(k L_{i}+b I_{N}^{i}\right) \theta(s)\right) d s \\
& \leq \int_{t_{k}^{i}}^{t}\left(\left\|z_{i}(s)\right\|+\left\|k L_{i}+b I_{N}^{i}\right\|\|\theta(s)\|\right) d s \\
& \leq \int_{t_{k}^{i}}^{t}\left(1+\left\|k L_{i}+b I_{N}^{i}\right\| \sqrt{\frac{\sigma \alpha}{\beta}}\right)\|z(s)\| d s .
\end{aligned}
$$

The last inequality comes from the theme of the eventbased strategy. Meanwhile, from the former theorem, with the control of the event-based strategy, the derivative of the Lyapunov function (12) is less than 0 when the consensus is not achieved and is equal to 0 when the consensus is achieved. In other words,

$$
\begin{aligned}
V(t) & =\frac{1}{2} \xi^{T}(t) \xi(t)=\frac{1}{2} z^{T}(t)\left(H^{-1}\right)^{T}\left(H^{-1}\right) z(t) \\
& \leq V(0)
\end{aligned}
$$

So one has $z^{T}(t) z(t) \leq 2 V(0) / \lambda_{\min }\left(H^{-1}\right)^{2}$. Thereby, one also has

$$
\begin{aligned}
& \left\|\theta_{i}(t)\right\| \\
& \leq\left\|\int_{t_{k}^{i}}^{t}\left(1+\left\|k L_{i}+b I_{N}^{i}\right\| \sqrt{\frac{\sigma \alpha}{\beta}}\right) \frac{\sqrt{2 V(0)}}{\lambda_{\min }\left(H^{-1}\right)} d s\right\| \\
& \leq \frac{\left(1+\left\|k L_{i}+b I_{N}^{i}\right\| \sqrt{\sigma \alpha / \beta}\right) \sqrt{2 V(0)}}{\lambda_{\min }\left(H^{-1}\right)}\left(t-t_{k}^{i}\right) .
\end{aligned}
$$

For brevity, we use a function $\Theta_{i}(t)$ to represent the right hand side of the above inequality. Note that, compared to the function $\Theta_{i}(t),\left\|\theta_{i}(t)\right\|$ requires longer time to increase from zero to $\sqrt{\sigma \alpha / \beta}\left\|z_{i}(t)\right\|$. That is, if, at time $\tau+t_{k}^{i}, \Theta_{i}(t)$ reaches $\sqrt{\sigma \alpha / \beta}\left\|z_{i}(t)\right\|$, the next event will happen at or later than the time $\tau+t_{k}^{i}$.

This implies that the time interval $t_{k+1}^{i}-t_{k}^{i}>\tau$, where $\tau$ satisfies

$$
\begin{aligned}
\frac{\left(1+\left\|k L_{i}+b I_{N}^{i}\right\| \sqrt{\sigma \alpha / \beta}\right) \sqrt{2 V(0)}}{\lambda_{\min }\left(H^{-1}\right)} \tau \\
=\sqrt{\frac{\sigma \alpha}{\beta}}\left\|\left(\tau+t_{k}^{i}\right)\right\| .
\end{aligned}
$$

When consensus is not achieved, $\left\|z_{i}\left(\tau+t_{k}^{i}\right)\right\|$ is not equal to zero. In that case, $\tau>0$ and therefore the event interval is positive.

Theorem 5 proves that the fully decentralized event-based control strategy is able to guarantee the consensus of the leader-follower networked system. However, from the event function (9), the continuous states of neighbors, that is, $z_{i}(t)$, $i \in N_{i}$, are required. This requirement is not compatible with the initial motivation which aims at saving communication. In the following part, another event function is designed such that only the sampled states of each agent's neighbors are required.

3.2. Event Function with State Measurement. In this part, an event function which uses the event measurement and the state of leader is proposed. By using this event function, the followers no longer require to continuously transmit their state to their neighbors. Instead, only when an event occurs, the corresponding follower will transmit its event measurement. Hence by using this event function, the communication frequency can be reduced. The event function $g_{i}(t)$ is given by

$$
g_{i}(t)=\left\|\theta_{i}(t)\right\|^{2}-c\left\|\eta_{i}\right\|^{2},
$$

where $c=\sigma \alpha /\left(2 \beta+2 \sigma \alpha k^{2}\|L\|^{2}\right)$, and $\eta_{i}$ is the $i$ th entry of vector $\eta \triangleq-k L \tilde{x}+B\left(\mathbf{1}_{N} x_{0}-x\right)$. Compared to the former event function (9), this new event function contains $\tilde{x}_{j}$ rather than $x_{j}$, which excludes the continuous communication among followers.

Theorem 7. Consider the leader-follower networked system as described in (1) and (2). Suppose that the topology among followers is connected, and there exists at least one follower that can receive information from the leader and conditions (10) and (11) are satisfied. Then the leader-follower consensus can be achieved if the events occur when the event condition (22) exceeds zero. Moreover, the event interval between two consecutive events is strictly positive.

Proof. By the definition of $\eta$, one has

$$
\begin{aligned}
\eta & =-k L \tilde{x}+B\left(\mathbf{1}_{N} x_{0}-x\right) \\
& =k L\left(\mathbf{1}_{N} x_{0}-\tilde{x}\right)+B\left(\mathbf{1}_{N} x_{0}-x\right)=k L \xi+k L \theta+B \xi \\
& =H \xi+k L \theta .
\end{aligned}
$$


Hence, $\|\eta\|^{2}=\|(H \xi+k L \theta)\|^{2}=\xi^{T} H^{T} H \xi+k^{2} \theta^{T} L^{T} L \theta+$ $2 k \theta^{T} L^{T} H \xi$. It follows that

$$
\begin{aligned}
\|\eta\|^{2} & \leq 2 \xi^{T} H^{T} H \xi+2 k^{2} \theta^{T} L^{T} L \theta \\
& =2 z^{T} z+2 k^{2} \theta^{T} L^{T} L \theta .
\end{aligned}
$$

Note that the event function implies that $\|\theta\|^{2} \leq c\|\eta\|^{2}$, which further indicates that $\|\theta\|^{2} \leq c\left(2 z^{T} z+2 k^{2} \theta^{T} L^{T} L \theta\right)$. By substituting $c=\sigma \alpha /\left(2 \beta+2 \sigma \alpha k^{2}\|L\|^{2}\right)$ into this inequality, one has $\|\theta\|^{2} \leq \sigma(\alpha / \beta)\|z\|^{2}$. Therefore, using the same Lyapunov function (12), via similar approach as that of Theorem 5, it is straightforward to show that $\dot{V}(t) \leq 0$ and $\dot{V}(t) \rightarrow 0$ as $t \rightarrow \infty$. This implies that the leader-follower consensus of the system is achieved. The exclusion of the Zeno-behavior can be shown via the same approach as that of Theorem 6 . We also let $\tau$ be the time that function $\Theta_{i}(t)$ costs for it growing from zero to $c\left\|\eta_{i}(t)\right\|$. Then $\tau$ is shorter than the time interval between two events, where $\tau$ satisfies

$$
\begin{gathered}
\frac{\left(1+\left\|k L_{i}+b I_{N}^{i}\right\| \sqrt{\sigma \alpha / \beta}\right) \sqrt{2 V(0)}}{\lambda_{\min }\left(H^{-1}\right)} \tau \\
=c\left\|\eta_{i}\left(\tau+t_{k}^{i}\right)\right\| .
\end{gathered}
$$

Therefore, we also have a strictly positive interevent interval when the leader-follower consensus is not achieved. Thus the Zeno-behavior is excluded.

\section{Simulations}

In this section, simulations are shown to illustrate the effectiveness of the proposed event-based control strategy. A leader-follower networked system consisting of 6 followers and 1 leader is adopted. We first give simulation for the decentralized event-based strategy and then change the event function into the event-measurement event function and run the simulation again. The Laplacian matrix of the followers' topology is

$$
L=\left[\begin{array}{cccccc}
1 & -1 & 0 & 0 & 0 & 0 \\
0 & 0 & 0 & 0 & 0 & 0 \\
-1 & 0 & 2 & -1 & 0 & 0 \\
0 & 0 & -1 & 2 & 0 & -1 \\
0 & 0 & 0 & 0 & 1 & -1 \\
0 & 0 & 0 & -1 & -1 & 2
\end{array}\right]
$$

The dynamics of the followers and the leader are governed by, respectively, (1) and (2), with $b=1$. We set $k=2$ and $k_{1}=0.1$ to satisfy conditions (10) and (11). At first, the control scheme (5) and the event function (9) are adopted. When the event function overpasses 0 , the follower samples its state and marks this time instant as the latest event instant. After that, the follower sends this sampled state together with the latest event instant to neighbors.

The state differences between each follower and the leader, as previously denoted using $\xi_{i}, i=1, \ldots, 6$, are

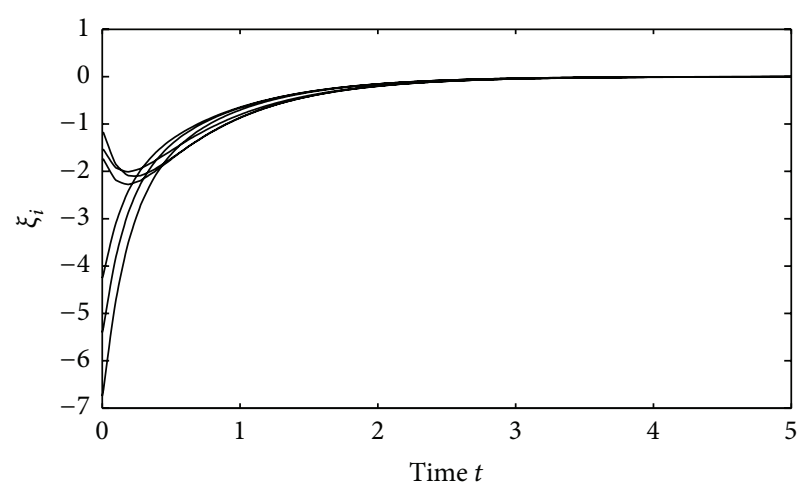

FIGURE 1: State difference between follower $i$ and the leader for $i=$ $1, \ldots, 6$.

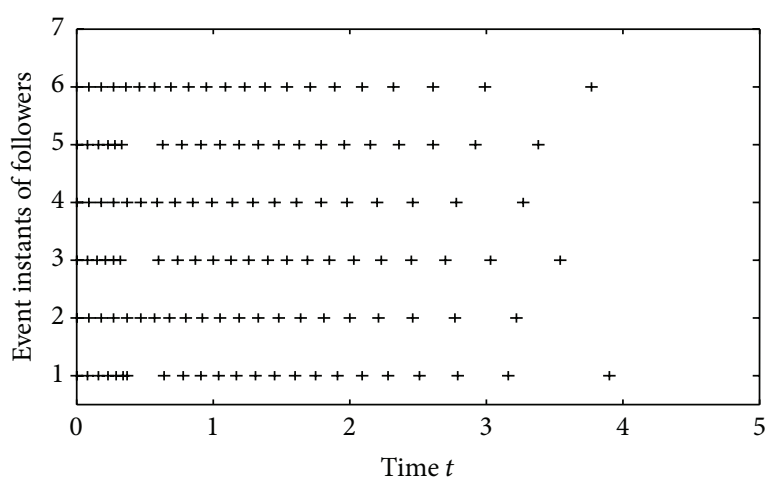

FIGURE 2: Event sequences for followers.

shown in Figure 1. As observed, the system reaches the leaderfollower consensus asymptotically. Figure 2 illustrates the event instants for each of the followers. It is shown that each agent triggers asynchronously. In order to inspect the motion of the event measurement, the evolution of $\left\|\theta_{6}(t)\right\|$ is shown in Figure 3. As observed, $\left\|\theta_{6}(t)\right\|$ is set to zero at the event instants and it grows until the event function (9) exceeds zero.

Now we use the second event function (22) that only utilizes the event measurement rather than the continuous state of neighbors.

Figure 4 illustrates the trajectory of $\xi_{i}, i=1, \ldots, 6$. As observed, with the event function (22), the system can reach the leader-follower consensus. The event sequences are shown in Figure 5 and the evaluation of the event measurement of follower 6 is shown in Figure 6.

From Figures 2 and 4, it is observed that the event function (22) generates less events than event function (9). This property can reduce the communication times of the system. In addition, from the comparison between Figures 3 and 6 , one can see that the event function (22) generates less events for follower 6 .

Remark 8. Theorem 6 only proves that when the consensus is not achieved the interval is positive rather than strictly lower bounded. In the future, the existence of the lower bound will be considered. After the consensus is achieved, for the worst case, the event-based control strategy will not cause 


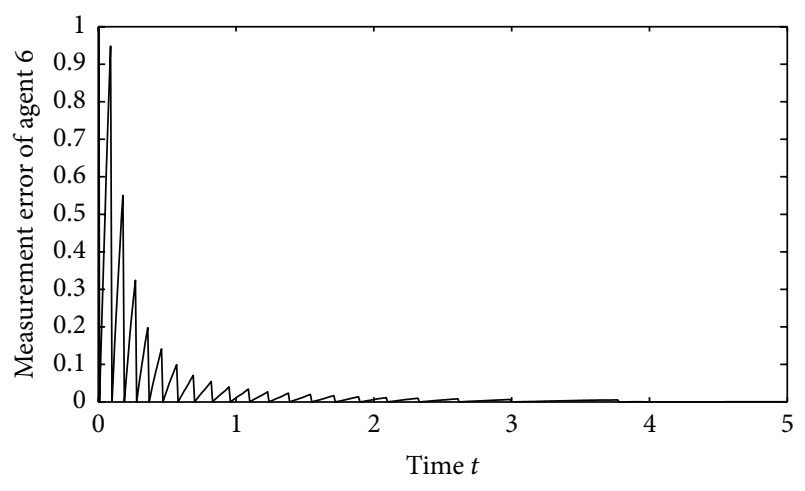

FIGURE 3: Event measurement of follower 6.

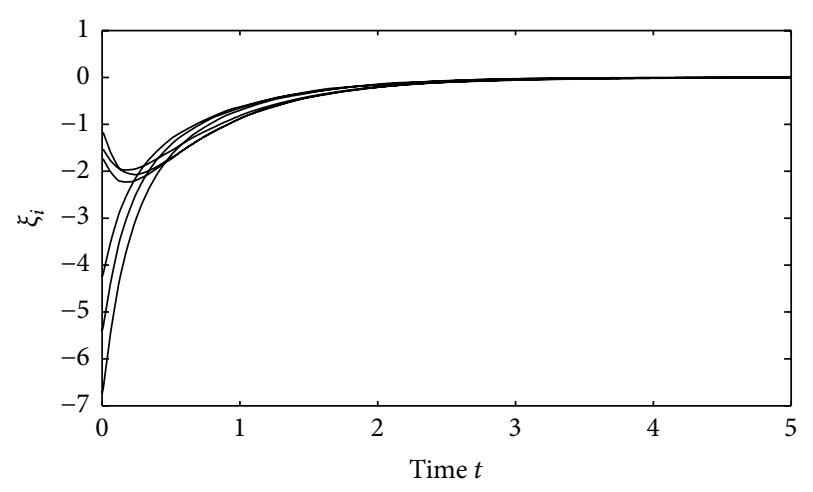

FIGURE 4: State difference between follower $i$ and the leader for $i=$ $1, \ldots, 6$.

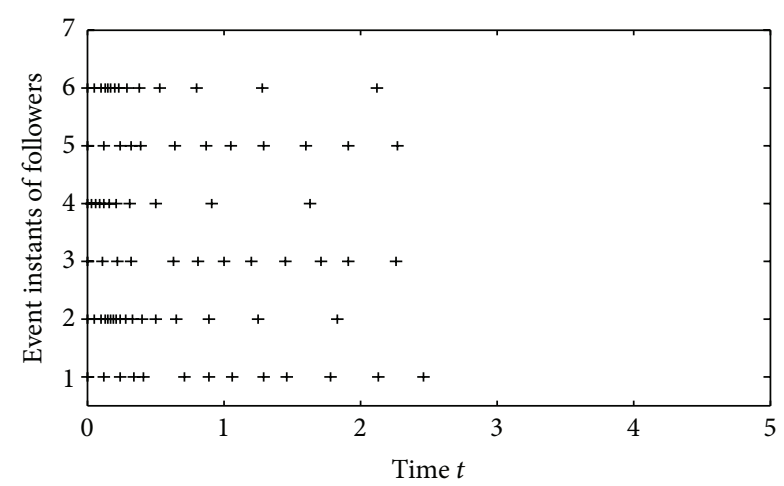

Figure 5: Event sequences for followers.

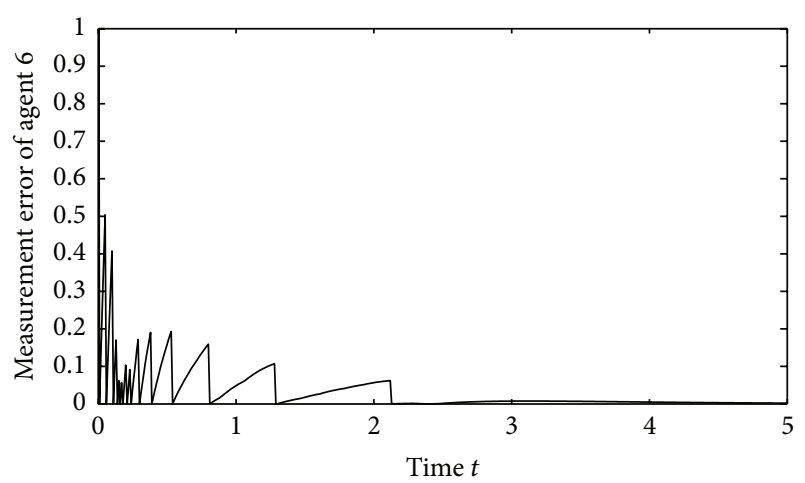

FIGURE 6: Event measurement of follower 6. more communications than continuous protocols. However, from the simulations, one can see that the event-based control strategy is effective and there is no Zeno-behavior.

\section{Conclusion}

We considered the consensus problem for leader-follower networked systems in this paper. An event-based control strategy associated with event function is utilized to control the followers in a decentralized manner. Furthermore, the event function is developed such that the continuous states of neighbors are no longer required. Instead of that, the event measurement is used, which is transmitted only at neighbors' event instant. It is shown that the proposed scheme is able to decrease the channel occupations and communication frequencies while achieving the leader-follower consensus for the systems using only the local information. The Zenobehavior is also analytically excluded for both cases. Simulations are implemented to show the effectiveness of the proposed strategies. Future work includes extending the proposed methods to higher order networked systems and studying other collective behaviors.

\section{Competing Interests}

The authors declare that they have no competing interests.

\section{Acknowledgments}

This work was supported in part by the National Natural Science Foundation under Grant 61203056, Jiangsu Planned Projects for Postdoctoral Research Funds 1501022C, Hunan Provincial Science and Technology Program under Grant 2015GK3022, and the Construct Program of the Key Discipline in Hunan Province.

\section{References}

[1] J. A. Fax and R. M. Murray, "Information flow and cooperative control of vehicle formations," IEEE Transactions on Automatic Control, vol. 49, no. 9, pp. 1465-1476, 2004.

[2] C. W. Reynolds, "Flocks, herds, and schools: a distributed behavioral model," Computer Graphics, vol. 21, no. 4, pp. 25-34, 1987.

[3] H. Zhao, S. Xu, and D. Yuan, "Consensus of data-sampled multiagent systems with Markovian switching topologies," Asian Journal of Control, vol. 14, no. 5, pp. 1366-1373, 2012.

[4] Y. Hong, J. Hu, and L. Gao, "Tracking control for multiagent consensus with an active leader and variable topology," Automatica, vol. 42, no. 7, pp. 1177-1182, 2006.

[5] W. Ren and R. W. Beard, "Consensus algorithms for doubleintegrator dynamics," in Distributed Consensus in Multi-Vehicle Cooperative Control: Theory and Applications, pp. 77-104, Springer, London, UK, 2008.

[6] Q. Song, J. Cao, and W. Yu, "Second-order leader-following consensus of nonlinear multi-agent systems via pinning control," Systems \& Control Letters, vol. 59, no. 9, pp. 553-562, 2010. 
[7] W. Yu, G. Chen, and M. Cao, "Some necessary and sufficient conditions for second-order consensus in multi-agent dynamical systems," Automatica, vol. 46, no. 6, pp. 1089-1095, 2010.

[8] Q. Ma and J. Lu, "Cluster synchronization for directed complex dynamical networks via pinning control," Neurocomputing, vol. 101, pp. 354-360, 2013.

[9] J. Li, W. Ren, and S. Xu, "Distributed containment control with multiple dynamic leaders for double-integrator dynamics using only position measurements," IEEE Transactions on Automatic Control, vol. 57, no. 6, pp. 1553-1559, 2012.

[10] W. Yu, W. X. Zheng, G. Chen, W. Ren, and J. Cao, "Second-order consensus in multi-agent dynamical systems with sampled position data," Automatica, vol. 47, no. 7, pp. 1496-1503, 2011.

[11] W. Chen, X. Li, and L. C. Jiao, "Quantized consensus of secondorder continuous-time multi-agent systems with a directed topology via sampled data," Automatica, vol. 49, no. 7, pp. 22362242, 2013.

[12] L. Cheng, Y. Wang, Z.-G. Hou, M. Tan, and Z. Cao, "Sampled-data based average consensus of second-order integral multi-agent systems: switching topologies and communication noises," Automatica, vol. 49, no. 5, pp. 1458-1464, 2013.

[13] H. Shen, J. H. Park, L. Zhang, and Z.-G. Wu, "Robust extended dissipative control for sampled-data Markov jump systems," International Journal of Control, vol. 87, no. 8, pp. 1549-1564, 2014.

[14] P. Tabuada, "Event-triggered real-time scheduling of stabilizing control tasks," IEEE Transactions on Automatic Control, vol. 52, no. 9, pp. 1680-1685, 2007.

[15] D. V. Dimarogonas, E. Frazzoli, and K. H. Johansson, "Distributed event-triggered control for multi-agent systems," IEEE Transactions on Automatic Control, vol. 57, no. 5, pp. 1291-1297, 2012.

[16] X. Yin, D. Yue, and H. Su, "Event-triggered tracking control for discrete-time multi-agent systems," IMA Journal of Mathematical Control and Information, vol. 31, no. 2, pp. 165-182, 2014.

[17] H. Yan, Y. Shen, H. Zhang, and H. Shi, "Decentralized eventtriggered consensus control for second-order multi-agent systems," Neurocomputing, vol. 133, pp. 18-24, 2014.

[18] W. Zhu, Z.-P. Jiang, and G. Feng, "Event-based consensus of multi-agent systems with general linear models," Automatica, vol. 50, no. 2, pp. 552-558, 2014.

[19] X. Meng and T. Chen, "Event based agreement protocols for multi-agent networks," Automatica, vol. 49, no. 7, pp. 2125-2132, 2013.

[20] G. Guo, L. Ding, and Q.-L. Han, "A distributed event-triggered transmission strategy for sampled-data consensus of multiagent systems," Automatica, vol. 50, no. 5, pp. 1489-1496, 2014.

[21] Z. Liu and Z. Chen, "Reaching consensus in networks of agents via event-triggered control," Journal of Information \& Computational Science, vol. 8, no. 3, pp. 393-402, 2011.

[22] X. Chen, F. Hao, and M. Shao, "Event-triggered consensus of multi-agent systems under jointly connected topology," IMA Journal of Mathematical Control and Information, vol. 32, no. 3, pp. 537-556, 2015.

[23] J. Hu, G. Chen, and H.-X. Li, "Distributed event-triggered tracking control of second-order leader-follower multi-agent systems," in Proceedings of the IEEE 30th Chinese Control Conference (CCC '11), pp. 4819-4824, Yantai, China, July 2011.

[24] D. Xie, D. Yuan, J. Lu, and Y. Zhang, "Consensus control of second-order leader-follower multi-agent systems with eventtriggered strategy," Transactions of the Institute of Measurement and Control, vol. 35, no. 4, pp. 426-436, 2013.
[25] B. Zhou, X. Liao, T. Huang, and G. Chen, "Leader-following exponential consensus of general linear multi-agent systems via event-triggered control with combinational measurements," Applied Mathematics Letters, vol. 40, pp. 35-39, 2015.

[26] T.-H. Cheng, Z. Kan, J. M. Shea, and W. E. Dixon, "Decentralized event-triggered control for leader-follower consensus," in Proceedings of the 53rd IEEE Annual Conference on Decision and Control (CDC '14), pp. 1244-1249, IEEE, Los Angeles, Calif, USA, December 2014.

[27] H. Li, X. Liao, T. Huang, and W. Zhu, "Event-triggering sampling based leader-following consensus in second-order multi-agent systems," IEEE Transactions on Automatic Control, vol. 60, no. 7, pp. 1998-2003, 2015.

[28] T.-H. Cheng, Z. Kan, J. M. Shea, and W. E. Dixon, "Decentralized event-triggered control for leader-follower consensus," in Proceedings of the 53rd IEEE Annual Conference on Decision and Control (CDC '14), pp. 1244-1249, Los Angeles, Calif, USA, December 2014.

[29] B. Zhou, X. Liao, T. Huang, and G. Chen, "Pinning exponential synchronization of complex networks via event-triggered communication with combinational measurements," Neurocomputing, vol. 157, pp. 199-207, 2015.

[30] C. Godsil and G. Royle, Algebraic Graph Theory, vol. 207, Springer, New York, NY, USA, 2001.

[31] X.-H. Wang and H.-B. Ji, "Leader-follower consensus for a class of nonlinear multi-agent systems," International Journal of Control, Automation and Systems, vol. 10, no. 1, pp. 27-35, 2012.

[32] J. H. Wilkinson, The Algebraic Eigenvalue Problem, vol. 87, Clarendon Press, Oxford, UK, 1965. 


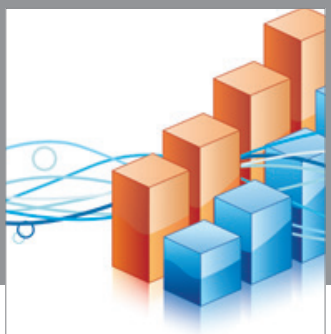

Advances in

Operations Research

vatem alat4

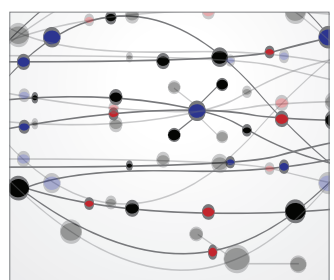

\section{The Scientific} World Journal
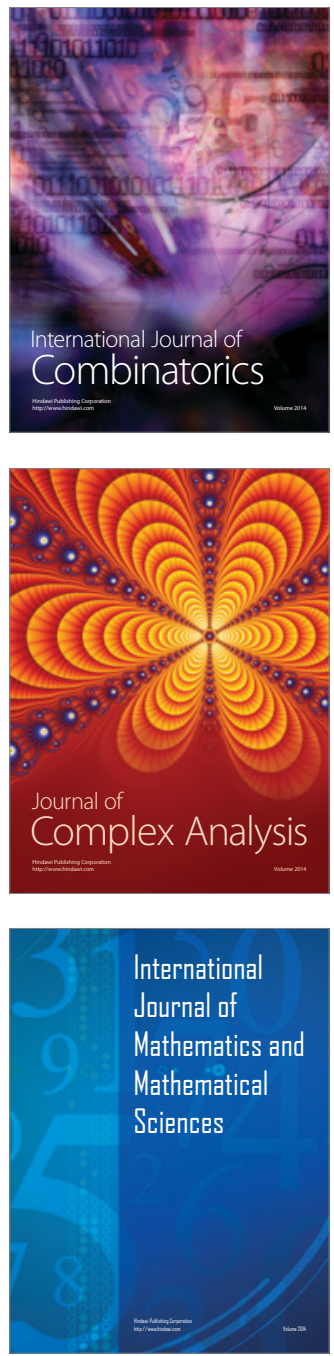
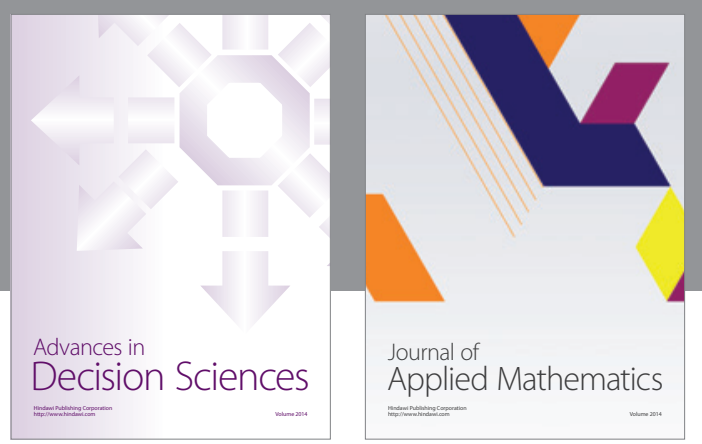

Algebra

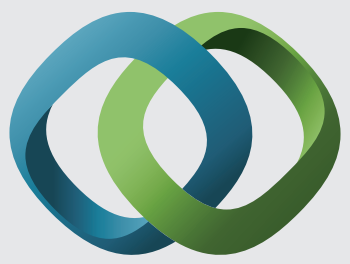

\section{Hindawi}

Submit your manuscripts at

http://www.hindawi.com
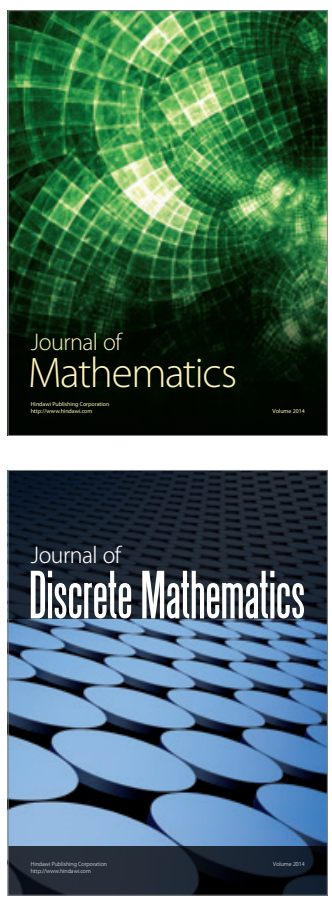

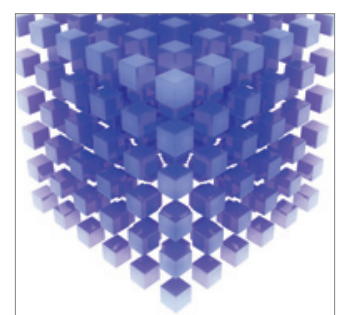

Mathematical Problems in Engineering
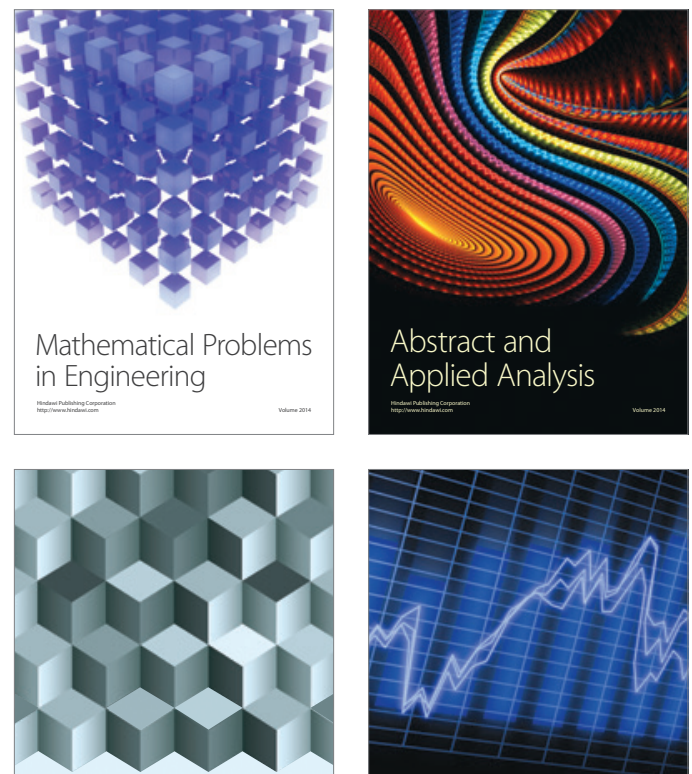

Journal of

Function Spaces

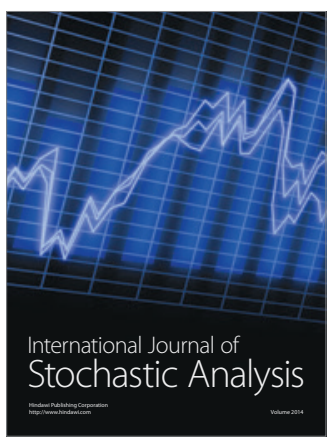

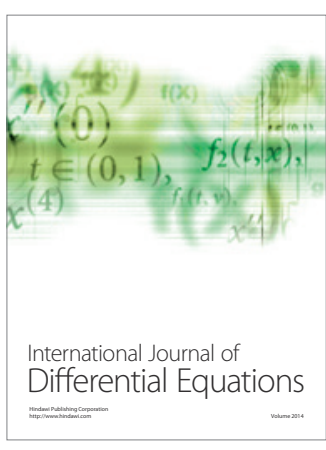
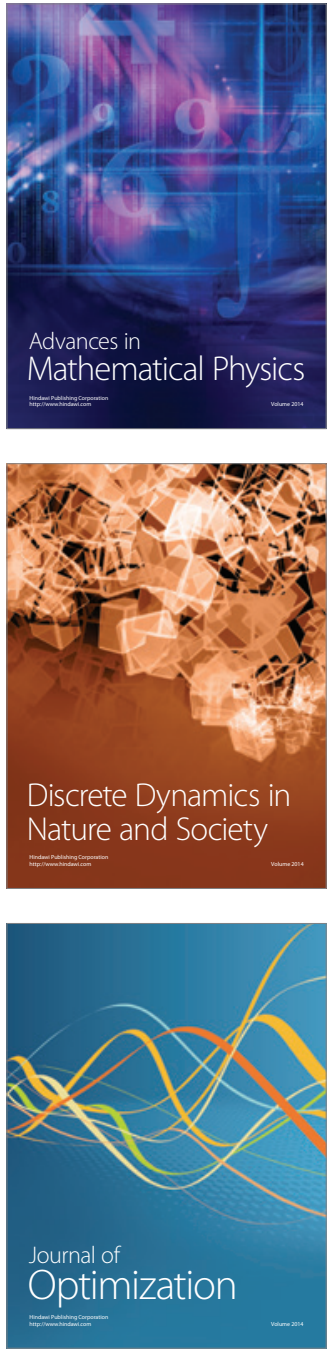